\title{
Computer-Assisted Learning of Chemical Experiments through a 3D Virtual Lab
}

\author{
Irene Luque Ruiz, Enrique López Espinosa, Gonzalo Cerruela García, and \\ Miguel Ángel Gómez-Nieto \\ Department of Computing and Numerical Analysis, University of Córdoba. \\ Campus Universitario de Rabanales, Edificio C2, Planta-3. E-14071 Córdoba, Spain \\ Phone: +34957 212082 Fax: +34 957218630 \\ \{mallurui, malloese, gcerruela, mangel\}@uco.es
}

\begin{abstract}
A model for the development of virtual chemical experiments is proposed in this paper. The model is named $E(V)=M+m-$ Experiment (Virtual) $=$ Materials + method - and is based on the definition of chemical experiments through the use of a set of materials which are manipulated following a method or protocol. The proposed $E(V)=M+m$ model has been implemented in a multimedia system, which is executed through a Web standard browser. The system developed allows the representation and subsequent building of chemistry experiments in virtual 3D worlds to any degree of complexity. This system integrates three main components: a complex object-relational database storing any kind of information relationship with the experiments (e.g. chemical substances, materials, apparatus, etc.), a VRML browser in charged to visualize the experiments, and a Java interface which is in charged to communicate the database with the 3D browser and the user.
\end{abstract}

\section{Introduction}

A great number of studies published showing the efforts investing by the chemical community to adopt the new computer technologies in the teaching of this discipline [1-7]. The published papers present teaching material like tutorials mainly aimed at secondary education [8-9], small-to-mid-sized software programs mainly directed to areas such as: periodic table information, balancing reactions, detailed composition, calculation of molecular masses, viewing molecular structures, interpretation of spectra, etc. [9-13] and laboratory software designed to simulate teaching lab experiments [14-16].

In this paper we proposed a model that permit avoid much of the existing inconveniences of the current chemical virtual labs and an environment suitable for the teaching of chemical experiments, as it offers the following features:

1. The proposed model permits a simple representation of the domain of the problem (the experiments). This model permits the inclusion of the teaching component, since content may be ordered and sequentialized for adaptation to both the student and the experiment. 
2. There not exists any limitation of the experiments that can be defined. Experimental content can be reusable, allowing its partial or complete use in the building of new experiments.

3. Chemistry experiments can be performed at varying levels of complexity, in line with different educational grades.

4. The system may be executed in any platform. A standard Web browser is used as interface between the system and the user.

5. The 3D representation of the experiments in a VRML world facilitates the learning of its content by the user.

6. The integration between a complex database, a Java interface and the 3D world permit the full communication between the user and the systems during the performance of the experiments.

The paper first describes the objectives of the study, and then in Section 2 provides a description of the proposed model. The description includes specification of the structure and content of experiments in an object oriented model, allowing both the classification of knowledge in learning blocks and its sequencing in the teaching process. Section 3 describes the operating prototype, closing with a discussion of the project that mentions future projects and enhancements currently under progress.

\section{Representing Virtual Chemical Experiments}

The model proposed in the development of chemical virtual experiments is based on the supposition that a virtual experiment may be described by a method, which uses a set of materials. Running a virtual experiment, performed in an interactive computer environment using a system of dialogs, the user may experience a 3D presentation simulating a chemistry experiment. Each experiment takes place in a virtual environment containing all the materials with which the user might interact the virtual laboratory. Labs may be shared by multiple experiments and may also be built using different technologies and software, so user interaction will depend on the technology employed to build them.

Each virtual experiment has an objective and is thus accompanied by descriptive content aimed at delivering knowledge for the user to learn. While the nature and extent of the experimental content may be highly varied, presentation and user interaction is independent of the experiment.

Each experiment has a unique associated method. A method is a precise description of the tasks and resources used in the experiment. Each method comprises structure and content. The structure of the method lists the processes to be carried out during the experiment, while the content includes all necessary information and the way in which this is manipulated therein.

The method's structure is made up of a sequence of phases. Each phase involves a global task or activity performed during the experiment, which may be broken down into further elementary tasks called stages.

This structural breakdown of an experiment, in which different knowledge blocks are organized chronologically in a working network, is a key factor in user learning $[1,17]$. 


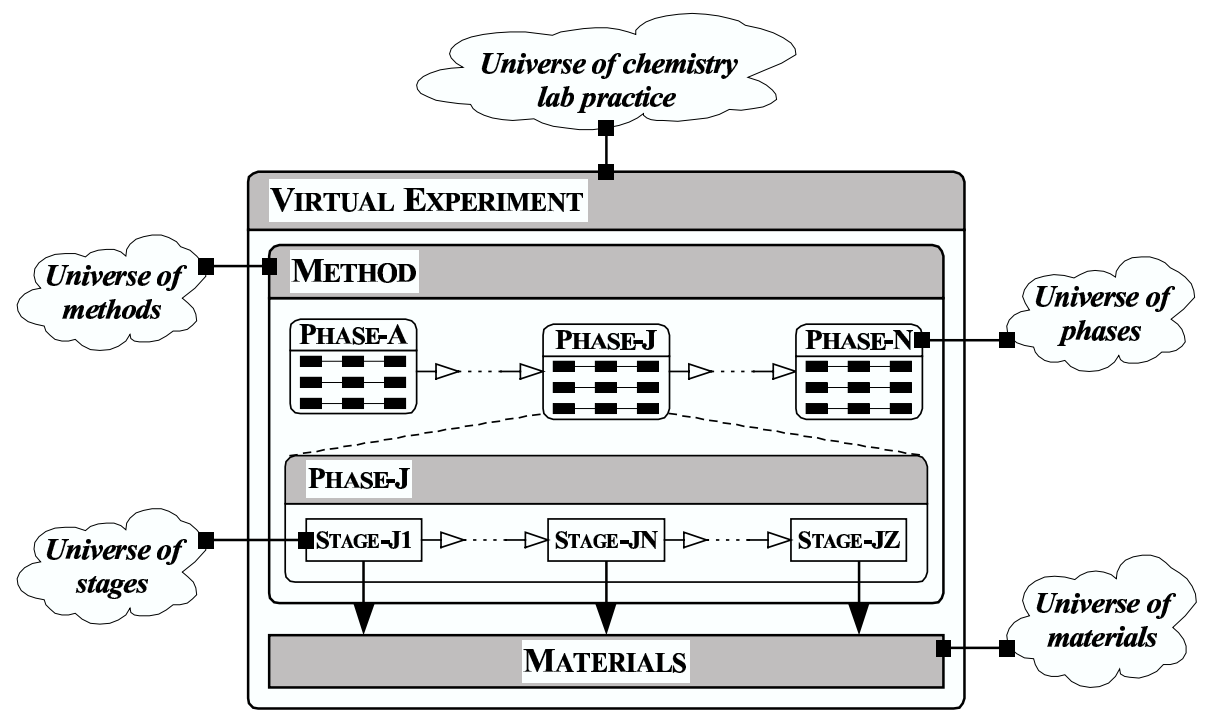

Fig. 1. Context diagram of the $E(V)=M+m$ model

Figure 1 shows a context diagram of the model $E(V)=M+m$. It may be seen that a virtual experiment represents the knowledge of a laboratory experiment through a method structured in phases, which in turn are divided into stages where the materials are manipulated. These structural elements of experiments are extracted from a general universe, enabling them to be reused in the same or indeed in a different experiment.

Let us now examine the structural elements of the model using a bottom-up approach, according to complexity.

\subsection{Stages}

Stages are basic tasks in the structural description of an experiment. Depending on the level of detail required for the description of an experiment, a task may vary from a simple manipulation such as "Obtain a pipette" to one like "Obtain a pipette, fill it, and empty the contents into an Erlenmeyer flask".

Stages imply manual or automated processes carried out in the lab and involving some laboratory material. They are distributed throughout the experiment, grouped in Phases. Within a phase, stages comprise a sequence of tasks that may be described by one or more directed graphs.

Take a phase- $k$ consisting of "Pour $10 \mathrm{ml} \mathrm{HCl}$ from the stock bottle into an Erlenmeyer flask". This phase may be defined by the following stages:

PKS1: Obtain a $10 \mathrm{ml}$ pipette from the rack

PKS2: Open the bottle of concentrated $\mathrm{HCl}$.

PKS3: Pipette $10 \mathrm{ml} \mathrm{HCl}$ from the bottle.

PKS4: Take the Erlenmeyer flask from the shelf and place on the bench.

PKS5: Empty the pipette's contents into the Erlenmeyer flask 
PKS6: Place the pipette in the sink.

PKS7: Close the bottle of concentrated $\mathrm{HCl}$.

Figure 2 contains a transition diagram [18] showing the different routes that could be taken to perform phase- $k$ (for a clearer description, the user is assumed to be reasonably skilled). Again in Figure 2, stages are represented by labeled boxes: shaded boxes show stages that could be the start of phase-k (S1,S2, S4), and the arrows indicate the transitions allowed between stages. If when performing phase- $k$ the user chose to begin at stage $S 1$, the next permissible stage would be $S 2$ or $S 4$. If the user opted to follow the transition to $S 2$, the next stage could be either $S 3$ or $S 4$.

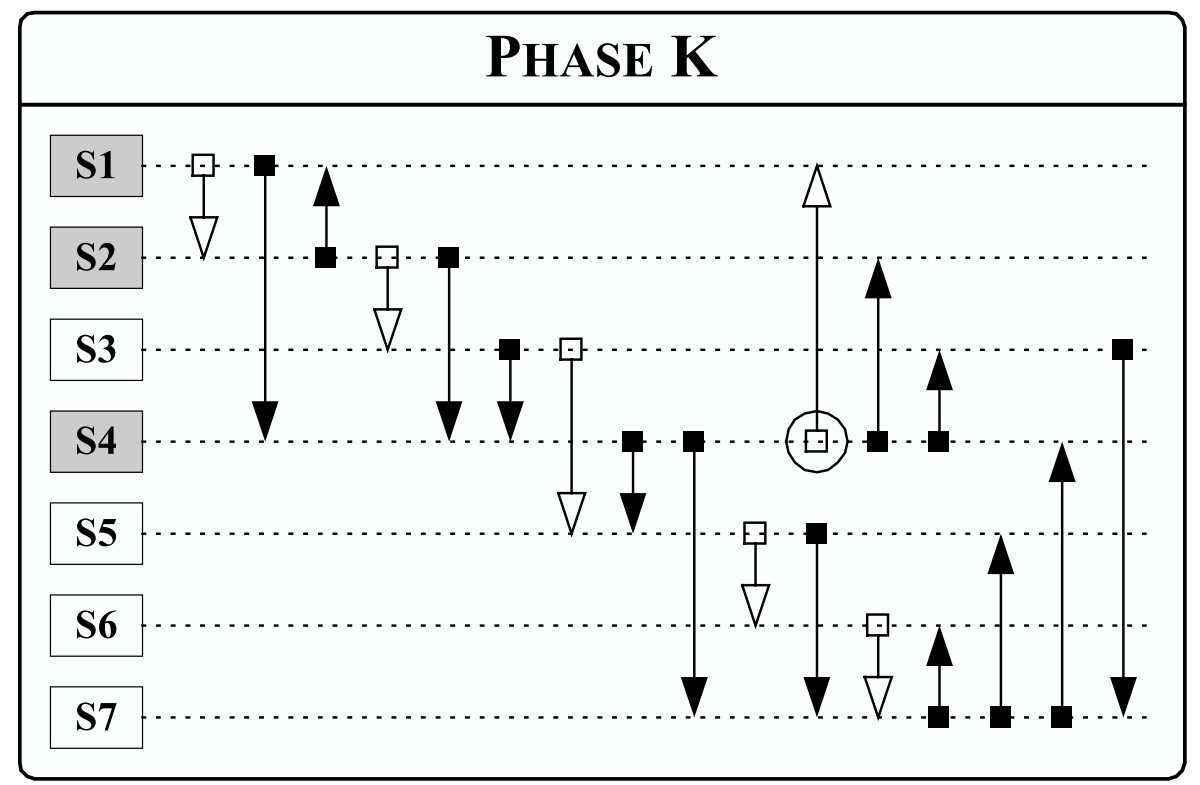

Fig. 2. Diagram showing permitted transitions for phase-k

If the transition to $S 3$ were chosen, the next move would have to be to $S 4$, since getting to $S 5$ would suppose a transition prior to $S 3$, that is, an Erlenmeyer flask must be present.

Alternatively, if the user had decided upon a transition to $S 4$, the next move must be to $S 3$, since moving on to $S 5$ would require a transition prior to stage $S 3$, that is, the pipette has to be filled, and moving to $S 7$ would mean that not all the transitions to all the stages of phase- $k$ could be completed by any of the existing routes.

Thus, if phase-k is started at $S l$ it can be performed suitably or correctly by the system using any of the following routes:

\begin{tabular}{|c|c|c|}
\hline R1: & S1, S2, S3, S4, S5, S6, S7 & R2: S1, S2, S3, S4, S5, S7, S6 \\
\hline R3: & S1, S2, S3, S4, S7, S5, S6 & R4 S1, S2, S4, S3, S5, S6, S7 \\
\hline R5: & S1, S2, S4, S3, S5, S7, S6 & R6: S1, S2, S4, S3, S7, S5, S6 \\
\hline R7 & S1, S4, S2, S3, S5, S6, S7 & R8: S1, S4, S2, S3, S5, S7, S6 \\
\hline R9 & $\mathrm{S} 1, \mathrm{~S} 4, \mathrm{~S} 2, \mathrm{~S} 3, \mathrm{~S} 7, \mathrm{~S} 5, \mathrm{~S} 6$ & \\
\hline
\end{tabular}


White arrows in Figure 2 show the permitted route that is considered, on defining phase- $k$, as the most recommendable and which will be taken into account by the system for the automatic execution of this phase without user intervention.

At each stage at least one Material is required for manipulation. The use of a material on occasion implies the presence of other materials, as shown by PKS3 where a pipette is used - thus implying the existence of the bottle of concentrated $\mathrm{HCl}$. Manipulation of material implies:

1. The presence of the material as well as those materials thereby affected.

2. A condition where the material manipulated and any other intervening material are in such a state that the manipulation may be successfully performed.

In the same foregoing example of $P K S 3$ the bottle of $\mathrm{HCl}$ must be open to allow the pipetting of $10 \mathrm{ml}$ (a prior transition to $P K S 2$ has taken place) and the pipette has already been obtained (a prior transition to $P K S 1$ has occurred).

Thus, Figure 2 shows all the possible transitions that represent manipulation processes involving phase- $k$. However, from any given stage only some of these transitions will be allowed for the performance of phase- $k$, depending on the transitions previously carried out and therefore on the current state of the material.

The permitted routes for the user to perform a generic phase- $n$ will be those that:

1. Run through, with no repetition, all of the stages comprising phase-n.

2. For any given stage perform only those transitions that do not violate the priorities existing between the stages of phase- $n$, as defined by the state of the material.

\subsection{Phases}

Phases are global elements in the task network that detail a method and group together stages, amounting to a general task. Regarding an experiment, a phase is an activity that leads to an elementary step in the experiment and in which a recognizable, measurable result is produced. Like stages, phases may be defined to varying degrees of abstraction and thus represent experimental activity at different levels of complexity.

\subsection{Methods and Experiments}

Methods describe the structure and chronological route of activities as occurring in the course of an Experiment. A method is an abstract sequence of procedures representing the tasks to be carried out, the material to be used and its manipulation in order to successfully perform a chemistry experiment. Since phases and stages may be described at various levels of abstraction, one laboratory experiment can be described by different methods at various levels of complexity.

The term "experiments" is used to describe practical laboratory experiments whose operational technique and underlying knowledge are to be passed on to the student through a virtual environment.

In the $E(V)=M+m$ model, an experiment is defined as the use of a method to manipulate a set of Materials. Thus each experiment has an associated method that is described by the phases and stages in which the materials are employed. Furthermore, 
an experiment has an associated virtual world containing the materials to be used and where the activities described in its associated method are carried out.

\subsection{Materials}

Materials represent the physical elements recognized in the virtual world or laboratory associated with an experiment and which can be used therein.

The materials category embraces a great variety of laboratory items, differentiated by their properties and associated functionalities. Hence, the context of material includes such diverse items as chemicals (e.g. existing products), furniture (e.g. lab benches), material (e.g. glassware) or instruments (e.g. pH meters).

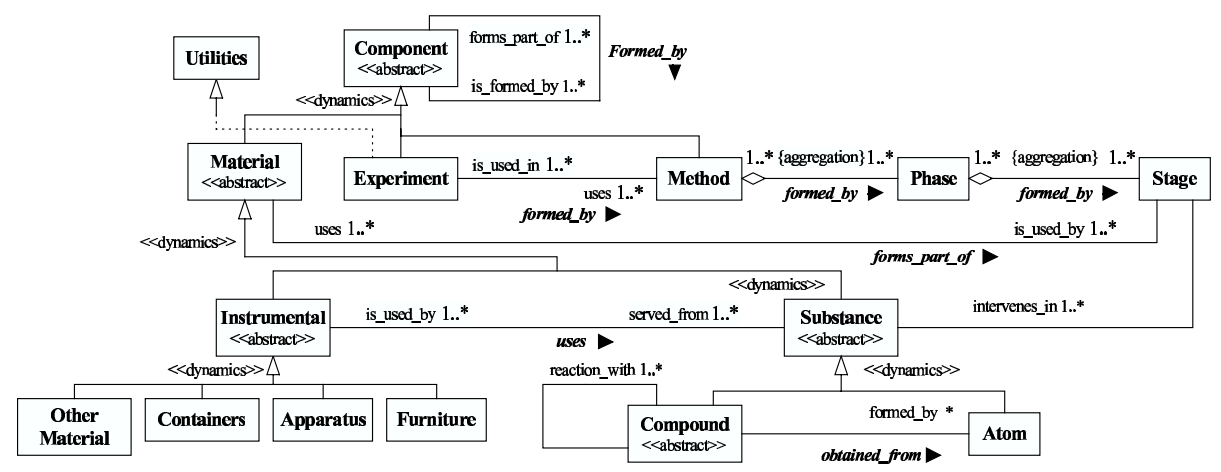

Fig. 3. Class diagram of the proposed model

\subsection{Modeling Virtual Experiments}

We was performed the implementation of the proposed model using the objectoriented paradigm, and complies with the UML standard [19]. Using the object model, a system can be described by a set of objects listing the properties and behavior of the system and each of its component parts.

The class diagram in Figure 3 shows the main entities of the static model representing the $E(V)=M+m$ model. A Component is considered to be any object existing in the system, and may in turn be formed by other components. The Component class is an abstract generalization class. By the term "abstract" we mean that objects belonging to the generalization class must be refined to one of the specialization classes of which it is composed.

A series of components is constructed, stored in a library and then used by a determined method, in a determined form and following a fixed timeline for the performance of an experiment, which in itself may be another component. A few examples of the objects included in this class would be: a decanting method, a precipitation flask, an experiment for the titration of $\mathrm{NaOH}$ with $\mathrm{HCl}$, filter paper, a funnel, lab scales, etc. 


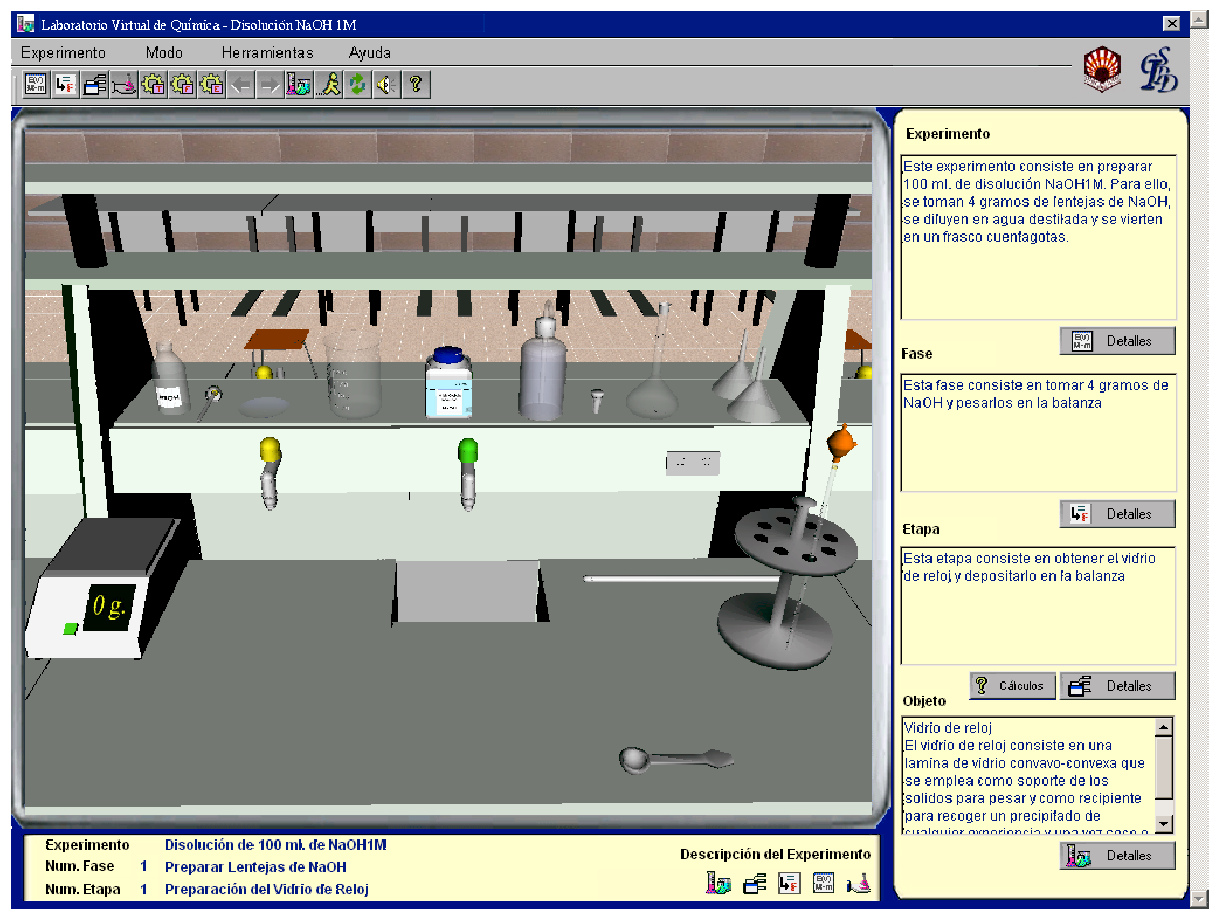

Fig. 4. An example of the interface of the system

\section{Implementation of the model}

An operational prototype of the proposed model has been built and is currently undergoing tests. It has been developed using the Java programming language, which is platform-independent and should run on any computer. The user interface has been created using Java applets that can be instantiated on most of the currently available browsers.

The Oracle 8i.X database manager handles information. The object-relational features of Oracle 8i.X permits the correct implementation of the class model described in the previous section. Moreover, this DBMS allows the storage of Java and VRML procedures in the very nucleus of the database alongside SQL procedure, in addition to storing media attributes like images and files in the definition of the table structure, which facilitates and enhances the development and performance of procedures [20,21].

The virtual world (the labs) and its components (materials) were developed under VRML 2.0, along with utilities to permit the graphical construction of these elements and their generation in VRML format [22,23].

The Figure 4 shows an example of the interface of the system. The Java interface coordinates the execution of the system, and it is in charged of the communication 
with the user, showing all information about of the evolution of the user-system interaction, which is carried out in a VRML virtual world.

\section{Discussion}

The proposal detailed in this paper is based on the use of virtual laboratories to provide extra support in the teaching of chemistry laboratory experiments. The proposal is based on $E(V)=M+m$ model that permits the structuring of knowledge in chunks of information describing basic stages in laboratory usage. The grouping of these stages in phases - both stages and phases are held in a set of permitted sequences - allows definition of the method or protocol for a lab experiment to be performed.

This structure, defined using a set of transitions that are allowed on the basis of the state of the materials employed, offers the possibility of the user carrying out an experiment in any permissible order of phases and stages and is thus not restricted to just one preset sequence, as is the case with other existing systems; moreover, the system can "advise" on the most suitable sequence and give feedback on the user's errors, a feature that adds greatly to its educational value.

The model has been implemented in an operational object-oriented prototype using the latest software technology (Oracle 8i, Java and VRML), bestowing the system with the set of properties required by authoring systems for the construction of intelligent tutorial systems; amongst these features are the reuse of software, easy integration of other components and a user-machine interface suited to the needs of assisted learning.

The authors are currently working to improve and expand several of the components of the subsystems that should form part of an ITS [24-26]. Additionally, work is underway on the definition of a suitable ontology for the construction of a dialog system between the user (student) and the system, such that through the use of an explanatory system running in parallel with the experiment, the student may both learn and be given explanations of conceptual and procedural aspects of the task in hand. In the present system this aspect is managed by static explanations held in .doc and. $\mathrm{html}$ files that are delivered to the user either on request or automatically, as might happen when some kind of exception arises.

\section{References}

1. Cloete, E., Electronic Education System Model., Comput. Educ., 36 (2001) 151-170

2. Lagowski, J.J. Chemical Education: Past, Present, and Future. J. Chem. Educ. 75 (1998) 425-436

3. Seal, K.C., Przasnysk, Z.H., Using The World Wide Web for Teaching Improvement., J. Chem. Inf. Comp. Sci., 36 (2001) 33-40

4. Warr, W.A., Communication and Communities of Chemistry, J. Chem. Inf. Comp. Sci., 38 (1998) 966-975

5. Borchardt, J.K., Improving the General Chemistry Laboratory, wwwchenweb.com/alchem2000/news/nw-000922-edu.html 
6. Jurs, P.C., Computer Software Application in Chemistry. Second Edition, John Wiley \& Sons Inc., New York, (1996)

7. Rzepa, H.S., Internet-Based Computational Chemistry Tools. In Enclyclopedia of Computational Chemistry. Wiley, London, (1998)

8. (a) marian.creighton.edu/ ksmith/tutorials.html, (b) www.emory.edu/CHEMISTRY/ pointgrp, (c) www.chem.uwimona.edu.jm:1104/chemprob.html, (d) www.chemsoc. org.golbook

9. (a) chem.lapeer.org/Chem1Docs/Index.html, (b) www.geocities.com/CapeCanaveral/ 9687/index.html, (c) www.lanzadera.com/qgeum, (d) www.scripps.edu/ nwhite/ B/Download/, (e) jchemed.chem.wisc.edu/JCEsoft/Issues/ Series_SP/SP8/abs-sp8.html, (f) chem-www.mps.ohio-state.edu/ lars/ moviemol.html

10. Ivancinc, O., ChemPlus for Windows, J. Chem. Inf. Comp. Sci., 36 (1996) 919-921

11. Ivanov, A.S., Rumgantsev, A.B., Archakov, A.I., Education Program for Macromolecules Structure Examination, J. Chem. Inf. Comp. Sci., 36 (1996) 660-663

12. Yoshida, H., Mausura, H. MOLDA for Windows. A Molecular Modeling and Molecular Graphics Program using a VRML Viewer on Personal Computers. Journal of Chemical Software, 3 (1997) 147-156 (cssj.chem.sci.hiroshima-u-ac.jp/molda/download.htm)

13. Nussbaum, N., Rosas, R., Peisano, I., Cardenas, F., Development Intelligent Tutoring Systems Using Knowledge Structure. Computers and Education, 36 (2001) 15-32

14. Borman, S., Lab Systems Embrace the Web, Chem. Eng. News. (27th, January (1997) 2527)

15. (a) www.modelscience.com/products.html, (b) www.chemnews.com, (c) website/ lineone.next/ chemie/reviews.html, (d) www2.acdlabs.com/ilabs, (e) www.chemsw.com/ 10202.htm, (f) www.compuchem.com/dldref/formdem.htm, (g) www.ir.chem.cmu.edu/ irProject/applets/virtuallab/applet_wPI.asp

16. Rzepa, H.S., Tonge, A.P., VchemLab: A Virtual Chemistry Laboratory. The Storage, Retrieval and Display of Chemical Information Using Standard Internet Tools, J. Chem. Inf. Comp. Sci., 36 (1998) 1048-1053

17. Luger, G.F., Stubblefield, W.A. Artificial Intelligence: Solutions and Strategies for Complex Problem Solving. Addison-Wesley, Reading MA (1992)

18. Jacobson, I., Booch, G., Rumbaugh, J., The Unified Software Development Process, Addison-Wesley Longman Inc, USA (1999)

19. Rumbaugh, J., Jacobson, I., Booch, G., The Unified Modeling Language. Reference Manual, Addison-Wesley Longman Inc, USA (1999)

20. Cary Anderson, J., Loy Stone, Blake Manual de Oracle JDeveloper, McGraw-Hill/Oracle Press, Madrid (1999)

21. Dorsey, P., Hudicka, J.R., Oracle8: Principios de Diseño de Base de Datos Usando UML, McGraw-Hill, Madrid (1999)

22. Roehl, Bernie, Couch, Justin, Reed-Ballreich, Cindy et al. Late Night VRML 2.0 with Java, Ziff-Davis Press, EmeryVille, California, USA (1997)

23. Lea, R., Matsuda, K. Miyashita, K. Java for 3D and VRML Worlds, New Riders, Indianápolis, USA (1996)

24. Luque Ruiz, I; Cruz Soto, J.L.; Gómez-Nieto, M.A. Error Detection, Recovery, and Repair in the Translation of Inorganic Nomenclatures. 1. A Study of the Problem. J. Chem. Inf. Comput. Sci. 36 (1996) 7-15. 2. A Proposed Strategy. J. Chem. Inf. Comput. Sci. 36 (1996) 16-24. 3. An Error Handler. J. Chem. Inf. Comput. Sci. 36 (1996) 483-490

25. Luque Ruiz, I.; Martínez Pedrajas, C.; Gómez-Nieto, M.A. Design and Development of Computer-Aided Chemical Systems: Representing and Balance of Inorganic Chemical Reactions. J. Chem. Inf. Comput. Sci. 40 (2000) 744-752

26. Luque Ruiz, I; Gómez-Nieto, M.A. Solving Incomplete Inorganic Chemical Systems through a Fuzzy Knowledge Frame. J. Chem. Inf. Comput. Sci., 41 (2001) 83-99. 